\title{
ARTICLE
}

Clinical Study

\section{Relationships between tumour response and primary tumour location, and predictors of long-term survival, in patients with $R A S$ wild-type metastatic colorectal cancer receiving first-line panitumumab therapy: retrospective analyses of the PRIME and PEAK clinical trials}

Marc Peeters ${ }^{1}$, Timothy Price ${ }^{1,2}$, Julien Taieb ${ }^{3}$, Michael Geissler $^{4}$, Fernando Rivera ${ }^{5}$, Jean-Luc Canon ${ }^{6}$, George Pentheroudakis ${ }^{7}$, Reija Koukakis ${ }^{8}$, Peter Burdon $^{9}$ and Salvatore Siena ${ }^{10}$

BACKGROUND: Data from two trials of panitumumab in metastatic colorectal cancer (mCRC) were retrospectively analysed to investigate the effects of primary tumour location on early-tumour shrinkage (ETS) and depth of response (DpR), and identify factors predicting long-term survival.

METHODS: Patients with RAS wild-type mCRC from PRIME (NCT00364013) and PEAK (NCT00819780) were included. ETS was defined as $a \geq 30 \%$ reduction in the sum-of-the-longest-diameters of measurable target lesions at eight weeks. DpR was the maximum percentage change from baseline to nadir in patients with shrinkage. Univariate and multivariate logistic analyses of short- versus long-term survivor data were performed.

RESULTS: A total of $435 / 559$ (78\%) patients had left-sided disease. Of these, a higher proportion of patients treated with panitumumab versus comparator achieved ETS (PRIME: $62 \%$ vs. $36 \%$; PEAK: $58 \%$ vs. $41 \%$ ); median DpR was also higher with panitumumab (PRIME: $59 \%$ vs. $49 \%$; PEAK: $70 \%$ vs. $48 \%$ ). In pooled analyses of the studies, more patients with right-sided disease achieved ETS if treated with panitumumab than comparator (39\% vs. $29 \%$ ). Panitumumab treatment consistently predicted longterm survival.

CONCLUSIONS: First-line panitumumab was associated with improved ETS and DpR vs. comparator in patients with left-sided mCRC. ETS may identify a subgroup of patients with right-sided disease who might respond to panitumumab.

British Journal of Cancer (2018) 119:303-312; https://doi.org/10.1038/s41416-018-0165-z

\section{INTRODUCTION}

Colorectal cancer (CRC) is one of the leading causes of cancer deaths worldwide. ${ }^{1}$ Almost $50 \%$ of patients with CRC develop metastatic disease, with $25 \%$ possessing distant metastases at the time of diagnosis. ${ }^{2}$ Systemic treatments for metastatic CRC (mCRC) include conventional fluoropyrimidine-based chemotherapy and biological agents that target vascular angiogenesis or the epidermal growth factor receptor (EGFR). ${ }^{3,4}$ The latter group of targeted agents include the anti-EGFR monoclonal antibodies panitumumab and cetuximab. Patients whose tumours harbour mutations in RAS do not respond to EGFR-targeted agents, and may in fact experience inferior outcomes if such agents are combined with oxaliplatin-containing chemotherapy regimens. ${ }^{4-6}$ It is therefore essential that before anti-EGFR therapy is initiated in a patient with $\mathrm{mCRC}$, RAS wild-type (WT) status is confirmed. ${ }^{3,4}$ V600E BRAF mutations-present in $~ 10 \%$ of CRC tumours-are a negative prognostic marker in patients with $\mathrm{mCRC}$, although current evidence on whether these mutations are predictive of the response to anti-EGFR agents is inconclusive. ${ }^{3,4,7-9}$ Furthermore, unlike $R A S$, extended (non-V600E) BRAF mutations may not have the same clinical implications. ${ }^{10}$ Additional biomarkers that may impact on the response to EGFR-targeted therapies, but remain to

\footnotetext{
${ }^{1}$ Department of Oncology, Antwerp University Hospital, Wilrijkstraat 10, 2650 Edegem, Belgium; ${ }^{2}$ Queen Elizabeth Hospital/University of Adelaide, 28 Woodville Rd, Woodville South, SA 5011, Australia; ${ }^{3}$ Sorbonne Paris Cité, Paris Descartes University, Georges Pompidou European Hospital, 20 Rue Leblanc, 75015 Paris, France; ${ }^{4}$ Klinikum Esslingen, Cancer Center Esslingen, Hirschlandstraße 97, 73730 Esslingen am Neckar, Germany; ${ }^{5}$ Hospital Universitario Marqués de Valdecilla, Av. Valdecilla, 25,39008 Santander, Spain; ${ }^{6}$ Service d'Oncologie-Hématologie, Grand Hôpital de Charleroi, Avenue du Centenaire 73, 6061 Charleroi, Belgium; ${ }^{7}$ Department of Medical Oncology, loannina University Hospital, Leof. Stavrou Niarchou, 45500 loannina, Greece; ${ }^{8}$ Biostatistics, Amgen Ltd, Sanderson Road, Uxbridge UB8 1DH, UK; ${ }^{9}$ European Medical, Amgen (Europe) GmbH, Dammstrasse 23, 6300

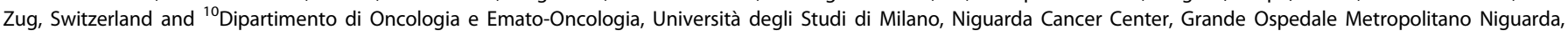
Piazza dell'Ospedale Maggiore, 3, 20162 Milan, Italy Correspondence: Marc Peeters (marc.peeters@uza.be)
} 
be confirmed, include human epidermal growth factor (HER) 2 and 3 and MET gene amplification.,11-13 For example, studies have indicated that HER2 activation substitutes for EGFR dependence in a subset of $\mathrm{mCRC}$ patients, and is therefore a potential negative predictor of benefit to anti-EGFR therapy. ${ }^{12}$

Another factor known to affect prognosis and treatment outcomes in $\mathrm{mCRC}$ is primary tumour location. Right-sided tumours are less prevalent and associated with a poorer prognosis than left-sided tumours. ${ }^{14-17}$ Right-sided tumours are also more frequently associated with mutations in BRAF, TGF $\beta R 2$ and PI3KCA. ${ }^{18,19}$ In contrast, amplification of EGFR and HER2, overexpression of EGFR ligands and chromosomal instability are more common in left- than right-sided tumours. ${ }^{18-20}$ According to the CRC Subtyping Consortium, right-sided tumours are more likely to be consensus molecular subtype 1 (CMS1), while left-sided tumours are predominantly CMS2. ${ }^{21}$

In exploratory analyses of two randomised controlled trials, PEAK and PRIME, tumour location was found to influence outcomes following treatment with the anti-EGFR monoclonal antibody, panitumumab. ${ }^{14}$ In the phase II PEAK study, patients with RAS WT left-sided disease treated with panitumumab plus modified (m)FOLFOX6 had numerically longer median PFS and median overall survival (OS) than patients treated with bevacizumab plus mFOLFOX6 (PFS: 14.6 vs. 11.5 months; OS: 43.4 vs. 32.0 months). In the larger phase III PRIME study, panitumumab plus FOLFOX4 significantly increased median PFS and median OS vs. FOLFOX4 alone in RAS WT patients with left-sided tumours (PFS: 12.9 vs. 9.2 months; OS: 30.3 vs. 23.6 months). For patients with right-sided tumours, however, no statistically significant differences in PFS or OS between panitumumab and the comparator arms were observed in either PRIME or PEAK, although no definitive conclusions could be drawn due to the relatively low number of these patients. ${ }^{14}$ In both trials, response rates were higher in the panitumumab arm vs. the comparator arm for both left- and right-sided RAS WT tumours. Analyses of other panitumumab studies showed that patients with RAS WT left-sided mCRC also benefit when this agent is provided as a second-line treatment. Meta-analyses of randomised controlled trials in mCRC have shown improved OS and PFS in RAS WT patients with left-sided tumours-but not in those with rightsided tumours - who were treated with EGFR-targeted antibodies plus chemotherapy, relative to chemotherapy alone or chemotherapy plus bevacizumab. ${ }^{22,23}$ In the same meta-analyses, however, numerical increases in ORR were observed in patients with right-sided tumours who received anti-EGFR treatment (as well as in those with left-sided tumours). ${ }^{22,23}$ These ORR findings indicate that doublet chemotherapy plus anti-EGFR therapy remains an option for patients with right-sided tumours in whom cytoreduction is the goal.

Here we report additional analyses of the PRIME and PEAK studies. The first set of these analyses were performed to further characterise the effect of primary tumour location and panitumumab treatment on previously untreated patients with RAS WT mCRC. Two newer measures of tumour response were considered: early-tumour shrinkage (ETS) and depth of response (DpR). Used increasingly in studies of $\mathrm{mCRC}$, ETS and DpR provide information on tumour shrinkage beyond that provided by the more traditional Response Evaluation Criteria In Solid Tumors (RECIST). ${ }^{24}$ ETS may also offer an early indication of sensitivity to treatment, ${ }^{25,26}$ while DpR reveals the maximum tumour shrinkage achieved. ${ }^{27}$ To date, there are limited data on these response assessments according to primary tumour location. The second set of analyses reported here were performed to build upon previous findings that primary tumour location influences outcomes in the first-line setting. Specifically, we aimed to identify other patient or disease characteristics that may predict good outcomes (longterm survival) in patients with $\mathrm{MCRC}$. To do this, we pooled data across treatment arms from PRIME and PEAK and compared characteristics between patients with, and those without, very extended survival. Preliminary results of these analyses have previously been presented in abstract form. ${ }^{28,29}$

\section{METHODS}

Study designs

Both PRIME and PEAK were randomised controlled trials that recruited patients with previously untreated mCRC. PRIME (ClinicalTrials.gov identifier: NCT00364013) was a phase III study that compared the efficacy and safety of panitumumab $6 \mathrm{mg} / \mathrm{kg}$ every two weeks (Q2W) plus FOLFOX4 with FOLFOX4 alone. ${ }^{30}$ PEAK (NCT00819780) was a phase II study of mFOLFOX6 combined with panitumumab $6 \mathrm{mg} / \mathrm{kg}$ Q2W or bevacizumab 5 $\mathrm{mg} / \mathrm{kg}$ Q2W. ${ }^{31}$ The current analyses focused on RAS WT mCRC and therefore only included data from patients whose tumours contained no mutations in KRAS or NRAS exons 2 (codons 12/ 13), 3 (codons 59/61) and 4 (codons 117/146). Patients were characterised as having BRAF mutations if mutations in BRAF exon 15 (at codon 600) were found. All procedures in PRIME and PEAK were carried out in accordance with the 1964 Helsinki declaration and its later amendments or comparable ethical standards, and with the ethical standards of the relevant institutional and/or national research committees. Signed informed consent was obtained from all patients. Separate consent was not required for these retrospective analyses.

\section{Analyses}

Impact of primary tumour location on outcomes. Information on primary tumour location was obtained from free-text surgery descriptions that were included in case report forms, and from original pathology reports. Primary tumours located in the caecum to transverse colon were coded as right-sided, while tumours located from the splenic flexure to rectum were considered leftsided. Baseline patient demographics and disease characteristics, duration of study drug exposure and post-progression (i.e. poststudy) anti-cancer therapy were summarised by study, study treatment and primary tumour location. The effects of primary tumour location on ETS and DpR were retrospectively assessed in each study and in pooled analyses of the two studies. ETS was defined as a reduction of $\geq 30 \%$ in the sum-of-the-longestdiameters of measurable target lesions at eight weeks after initiation of study treatment. A cut-off of $\geq 30 \%$ was selected as this is in line with RECIST criteria and has been associated with a similar pattern of benefit as a $\geq 20 \%$ cut-off. ${ }^{24} \mathrm{DpR}$ was calculated as the maximum percentage change from baseline to nadir in patients with tumour shrinkage. In patients with tumour growth or no change in tumour size, DpR was defined as the percentage change from baseline to progression if the patient subsequently progressed, or as 'missing' if the patient did not progress. Thus, $\mathrm{DpR}$ was positive if there was shrinkage, negative if there was growth and zero if there was no change. Other outcomes assessed in the single study and/or pooled analyses were PFS, OS, objective response rate and resection rate. A Cox proportional hazard model was used to calculate hazard ratios (HRs) and 95\% confidence intervals $(\mathrm{Cl})$ for PFS, OS and ETS. Significance was determined using the Wilcoxon rank-sum test. The Kaplan-Meier method was used to analyse PFS and OS by treatment, primary tumour location and ETS status.

Characteristics and response outcomes of long-term survivors. Long-term survivors were identified using two different definitions: patients with OS $\geq 45$ months or the $25 \%$ of patients with the longest OS. Given that OS in $\mathrm{mCRC}$ is reported to be $\geq 30$ months, 45 months was chosen as a reasonable cut-off for long-term survival. ${ }^{32,33}$ For validation, the $25 \%$ of patients with the longest OS were also analysed. Baseline demographic and disease characteristics, study exposure and outcomes of first-line therapy 
were summarised for each study. Based on the work of Köhne and colleagues, ${ }^{34}$ patients were retrospectively assigned a baseline prognostic score ('Köhne score') that classified subjects into high-, medium- and low-risk groups based on four baseline clinical parameters: Eastern Cooperative Oncology Group (ECOG) performance status, white blood cell count, alkaline phosphatase level and number of metastatic sites. ETS and DpR were also assessed, defined as above. Univariate logistic analyses were performed to descriptively assess the potential relationship of different covariates with long-term survival. The covariates included were sites of metastases, sex, treatment, ECOG performance status, BRAF status, primary tumour location, prior adjuvant chemotherapy, country, age, Köhne score and disease stage. A multivariate logistic analysis was then conducted using a stepwise model-building procedure with a $10 \%$ significance level for a covariate to enter or remain in the model. Patients who were censored before the cut-off date for the long-term survivor analyses were excluded from these analyses as it was not known if such patients were long- or short-term survivors (by either definition of long-term survival).

\section{RESULTS}

Impact of primary tumour location on outcomes

Patients, study drug exposure and post-progression anti-cancer therapy. As previously reported, ${ }^{14}$ there were 675 patients in the RAS WT populations of PRIME and PEAK. Among the 559 patients for whom primary tumour location could be determined, 435 (78\%) had left-sided tumours and a lower proportion, 124 (22\%), had right-sided tumours, reflective of the typical distribution in RAS WT populations. In both studies, patient demographics and disease characteristics at baseline were generally similar between patients with left- and right-sided disease, although those with right-sided disease were more likely to have BRAF mutations (Table 1). In PRIME but not PEAK, right-sided disease was more frequent in females than in males.

More patients with left-sided tumours than right-sided tumours received study drug for at least nine months. This was the case in both PRIME and PEAK (Table 1) and when data were pooled across the two studies $(159 / 435(37 \%)$ vs. $33 / 124(27 \%)$, respectively). Post-progression anti-cancer therapy was received by a greater proportion of patients with left-sided than right-sided disease (PRIME: $246 / 328$ (75\%) vs. $53 / 88$ (60\%); PEAK: $83 / 107$ (78\%) vs. $23 /$ $36(64 \%))$. In general, a similar trend was observed for each of the different treatments that were provided after progression (Table 1). Among patients treated with panitumumab, those with left-sided disease were more likely to receive post-progression anti-EGFR therapy than those with right-sided disease in both PRIME and PEAK. In contrast, among patients treated with comparator (FOLFOX4 alone in PRIME; mFOLFOX6 plus bevacizumab in PEAK), there were no differences in the proportion of patients with leftvs. right-sided disease who received post-progression anti-EGFR therapy (Table 1).

Outcomes-single study analyses. Table 2 shows, for PRIME and PEAK, the effects of panitumumab and comparator treatment on patient outcomes-including ETS and DpR-by primary tumour location. In patients with left-sided disease, ETS was achieved in a higher proportion of those who received panitumumab than those who received comparator (PRIME 62\% vs. 36\%; PEAK: $58 \%$ vs. $41 \%)$. In patients with right-sided disease, a similar proportion achieved ETS with panitumumab and comparator in PRIME (31\% in both treatment arms). In PEAK, however, a greater proportion of these patients experienced ETS with panitumumab than with comparator (55\% vs. $21 \%$ ). For patients with left-sided tumours, median DpR was higher with panitumumab than with comparator (PRIME: $59 \%$ vs. $49 \%$, PEAK: $70 \%$ vs. $48 \%$ ). The effects of treatment on DpR were less clear in patients with right-sided disease (PRIME: $37 \%$ vs. $50 \%$; PEAK: $50 \%$ vs. $45 \%$ ). In both studies, more patients with left- than right-sided disease underwent any resection and complete (R0) resection (Table 2 ).

Outcomes-pooled analyses of PRIME and PEAK. In pooled analyses of PRIME and PEAK, panitumumab improved PFS in patients with left-sided disease compared with comparator treatment (HR: 0.70, $95 \% \mathrm{Cl}: 0.58-0.86)$. OS was also improved with panitumumab vs. comparator in patients with left-sided disease (HR: $0.71,95 \% \mathrm{Cl}$ : 0.57-0.89; Fig. 1a). In patients with right-sided disease, the effects of panitumumab on PFS and OS relative to comparator were less clear (HR for PFS: $0.84,95 \%$ Cl: $0.58-1.23$; HR for OS: $0.90,95 \% \mathrm{Cl}$ : $0.61-1.32)$.

In a pooled analysis of both studies and all treatment arms, more patients with left- vs. right-sided disease experienced ETS $(214 / 435(49 \%)$ vs. $42 / 124(34 \%))$. In total, $39 \%(24 / 61)$ of patients with right-sided tumours who received panitumumab experienced ETS. In these patients, median PFS was 10.9 months $(95 \%$ $\mathrm{Cl}: 9.0-15.8)$ and median OS was 26.6 months (95\% Cl: 10.4-36.9). Among patients with right-sided disease who received comparator treatment, $29 \%(18 / 63)$ had ETS; these patients had a median PFS of 9.4 months $(95 \% \mathrm{Cl}: 6.2-16.6)$ and median OS of 23.6 months (95\% Cl: 9.8-34.5). Pooled analysis across studies and treatments showed that ETS was associated with improved PFS compared with ETS $<30 \%$, irrespective of primary tumour location (HR in left-sided disease: $0.57,95 \% \mathrm{Cl}$ : 0.46-0.70; HR in right-sided disease: $0.60,95 \% \mathrm{Cl}$ : 0.39-0.91). Similar results were seen for the impact of ETS on OS (HR in left-sided disease: 0.48 , 95\% Cl: 0.38-0.60; HR in right-sided disease: $0.58,95 \% \mathrm{Cl}$ : 0.38-0.89; Fig. 1b). Across both studies and all treatments, more patients with left- than right-sided disease underwent any resection $(65 / 435(15 \%)$ vs. $12 / 124(10 \%))$ or complete (R0) resection $(48 / 435(11 \%)$ vs. $5 / 124(4 \%))$.

Characteristics and response outcomes of long-term survivors Patients. A total of 612 patients were included in the long-term survivor analysis. Baseline demographics and disease characteristics for long- and short-term survivors (defined using the 45month or $25 \%$ cut-offs) are shown for each study in Supplementary Table 1. In both studies, long-term survivors defined using the 45-month cut-off were more likely than short-term survivors to have been treated with panitumumab, have an ECOG performance status of 0 , have BRAF WT $\mathrm{mCRC}$, have left-sided primary disease and have liver-only metastases. Similar trends were observed when long-term survival was defined using the $25 \%$ cut-off (Supplementary Table 1).

Outcomes during first-line therapy in long-term survivors. Responses of long- and short-term survivors (defined according to both cut-offs) are summarised in Table 3 . Using the $25 \%$ cut-off, median OS irrespective of treatment arm was 39.5 months in PRIME (120/475 patients) and 45.9 months in PEAK (34/137 patients). The $25 \%$ of patients with the highest OS were more likely, compared with short-term survivors, to have received study treatment for at least 9 months, achieved an objective complete or partial response, undergone any or complete resection, and experienced ETS (Table 3). These results were driven by those patients with left-sided tumours (Supplementary Table 2). Median DpR was also higher in these long-term survivors. Across PRIME and PEAK, 124 patients (21\%) survived for $\geq 45$ months (Table 3 ). In pooled analysis of the studies using the 45 -month cut-off, median DpR was $79 \%$ (interquartile range: $61-100$ ) in long-term survivors and $44 \%(22-61)$ in short-term survivors, with $68 \%(84 / 124)$ and $37 \%(175 / 478)$ of patients, respectively, experiencing ETS.

The covariates included in the descriptive univariate analysis, and the results of this analysis when the 45-month cut-off was used, are shown in Supplementary Table 3. In multivariate analyses, panitumumab treatment (vs. comparator) was the only factor that significantly predicted long-term survival in both PRIME 
Table 1. Baseline patient demographics and disease characteristics, study drug exposure and post-progression anti-cancer therapy by treatment and primary tumour location in PRIME and PEAK

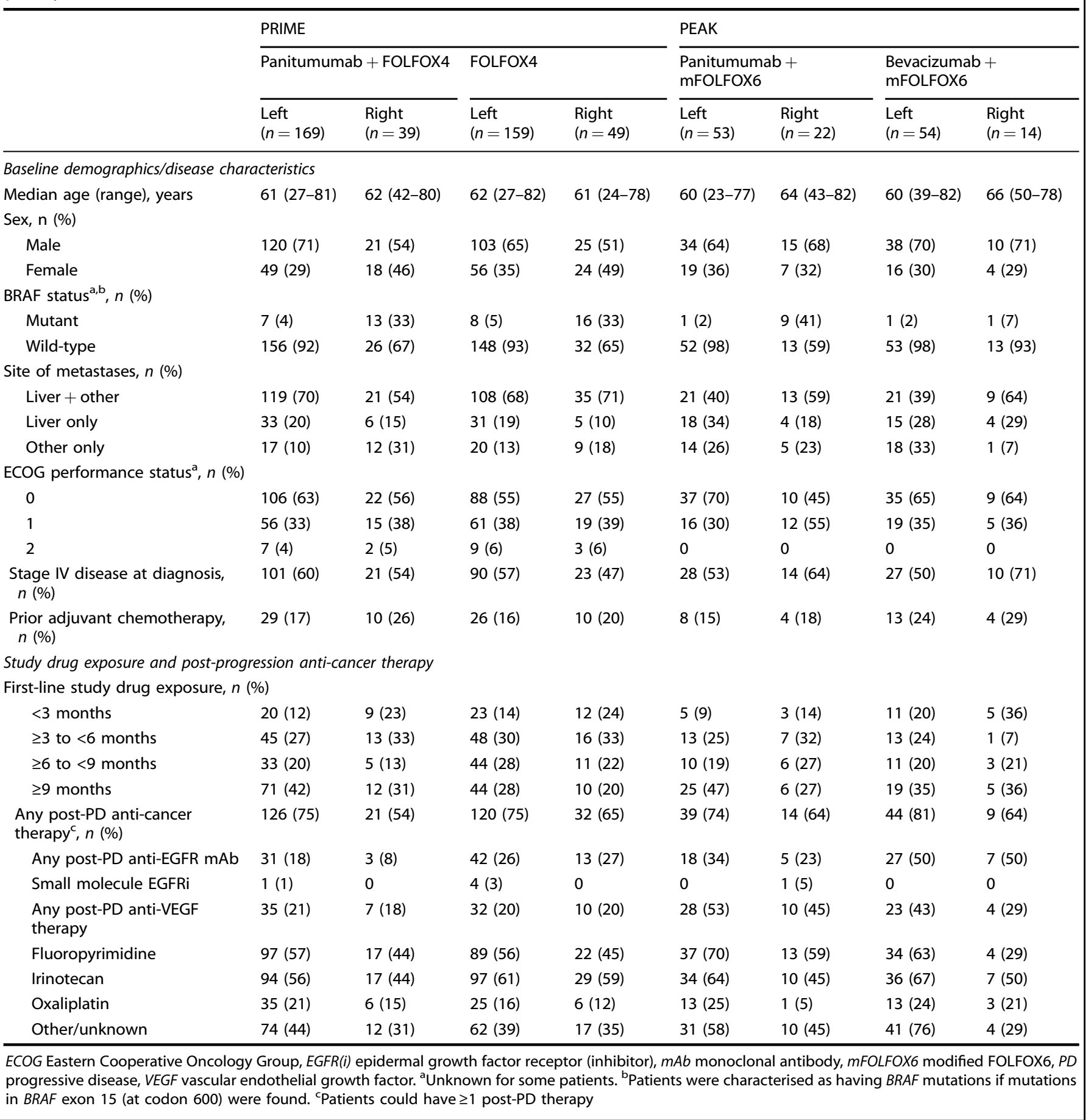

and PEAK according to both the 45-month cut-off (Table 4) and the $25 \%$ cut-off (data not shown). In PRIME, medium-risk Köhne score, liver-only metastases, ECOG performance status 0 (25\% cutoff only), BRAF WT status, study location in Western Europe, Canada or Australia (45-month cut-off only), age $<65$ years (45month cut-off only) and stage I disease (45-month cut-off only) were also significantly associated with long-term survival. In PEAK, female sex, left-sided primary tumour location, ECOG performance status 0 and prior adjuvant chemotherapy were associated with long-term survival (in addition to panitumumab treatment). In pooled multivariate analyses of PRIME and PEAK using the 45- month cut-off, panitumumab treatment again predicted longterm survival, along with low- and medium-risk Köhne score, $B R A F$ WT status and left-sided primary tumour location (Table 4).

\section{DISCUSSION}

We performed two new sets of retrospective analyses of PRIME and PEAK, two randomised controlled trials that recruited patients with previously untreated RAS WT mCRC. The main aim of the first set of analyses was to characterise the effects of primary tumour location and panitumumab treatment on patient outcomes in the 


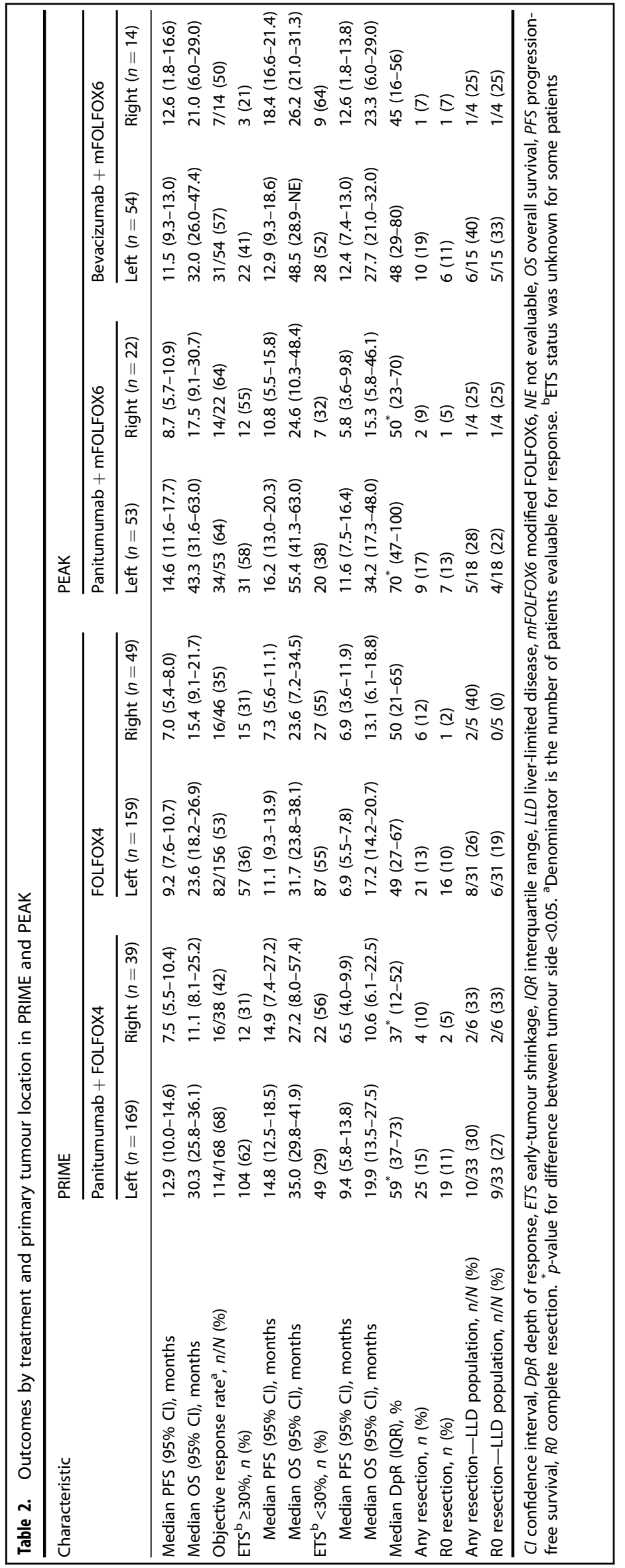



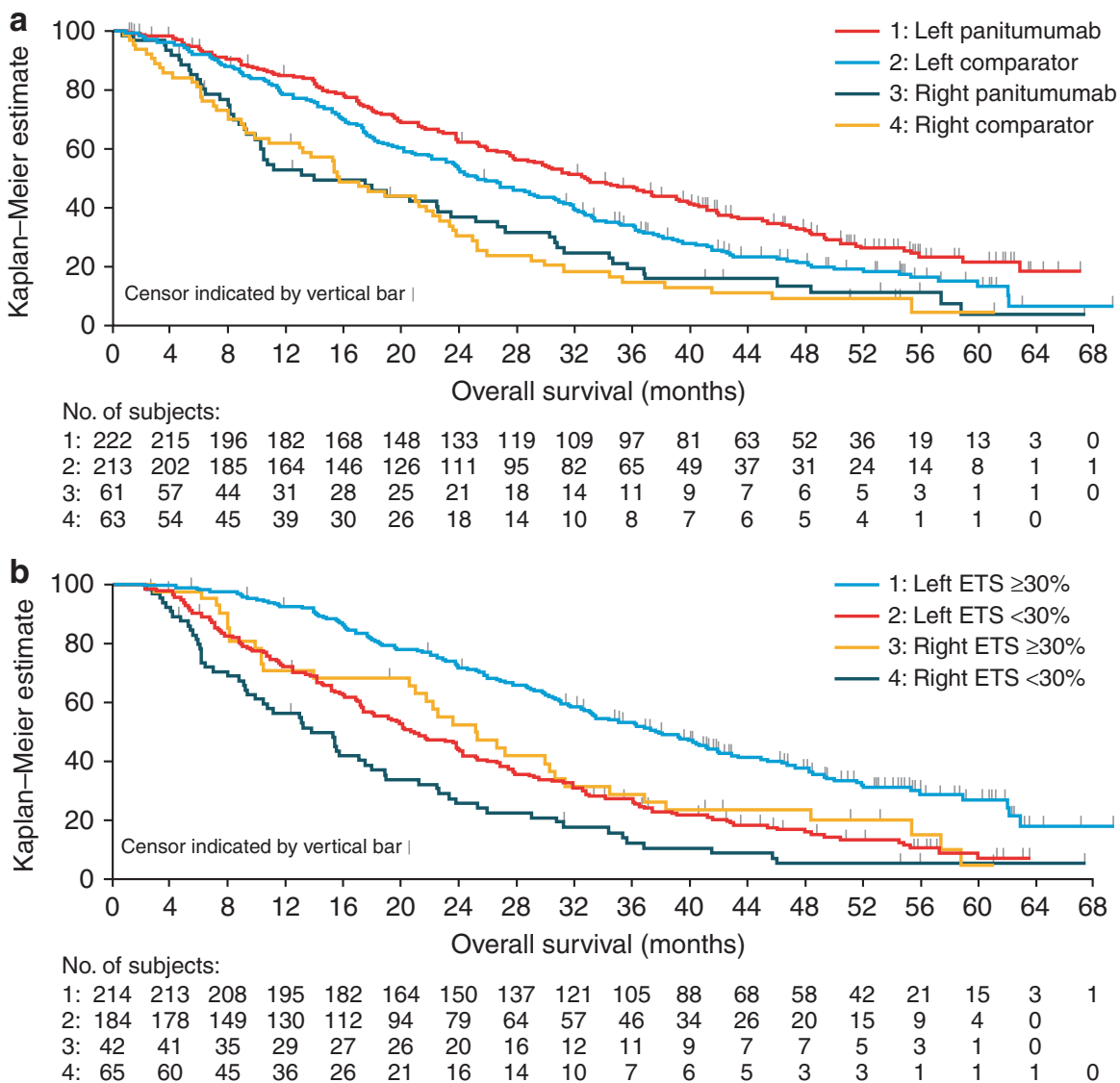

Fig. 1 Kaplan-Meier plots showing impact of a primary tumour location and treatment on OS, and b primary tumour location and ETS on OS in pooled analyses from PRIME and PEAK. ETS early-tumour shrinkage, OS overall survival

first-line setting, beyond those already reported. ${ }^{14}$ For patients with left-sided disease, our analyses suggest that in addition to its known beneficial effects on OS, PFS and other 'traditional' endpoints in this population, panitumumab may also improve outcomes relative to comparator treatment as measured by two newer endpoints, ETS and DpR. The data were less conclusive for patients with right-sided disease but suggest that ETS may identify a subgroup of these patients who might respond to panitumumab. The second set of analyses, which aimed to identify other patient or disease characteristics that may predict a favourable prognosis in patients with $\mathrm{mCRC}$, found that panitumumab treatment was the only factor that significantly predicted longterm survival in both trials.

Regarding the first set of analyses, individual assessments of PRIME and PEAK revealed that a higher proportion of patients with left-sided disease achieved ETS with panitumumab than with comparator. Median DpR was also higher with panitumumab vs. comparator in these patients in both studies. ETS, as well as DpR, provide information on tumour shrinkage over and above that provided by RECIST, specifically on the timing, depth and duration of response. As a result, they are increasingly being utilised as endpoints in mCRC studies. Achieving early and sustained tumour shrinkage is an important treatment goal in patients with $\mathrm{mCRC}$ as it may increase the chance of surgical resection, reduce chemotherapy-associated liver toxicity prior to resection and provide relief of tumour-related symptoms. ${ }^{26,35}$ That our pooled analysis of PRIME and PEAK showed superior survival outcomes with panitumumab vs. comparator in patients with left-sided disease was consistent with other reports. ${ }^{15-17}$ Overall, our findings in patients with previously untreated left-sided RAS WT tumours further confirm that anti-EGFR therapy is an appropriate choice for this population. However, the benefits of panitumumab treatment in the PEAK and PRIME studies were less evident in patients with right-sided disease, with DpR, OS and PFS findings, for example, being much less clear in this population. These results are consistent with past reports of an unclear benefit of anti-EGFR therapy in patients with RAS WT right-sided mCRC. ${ }^{14,16,22,23}$ Of note, however, objective response rates for right-sided disease have previously been shown to be higher for panitumumab vs. bevacizumab. ${ }^{14,22}$ Moreover, addition of panitumumab to FOLFOXIRI in the randomised phase II VOLFI trial resulted in high response rates in left- and right-sided RAS WT mCRC. ${ }^{36}$ It seems pertinent to ask then if there is a subgroup of patients with right-sided disease who should be considered for anti-EGFR therapy and, if so, how they can be identified. With these questions in mind, it is of interest that across the two studies reported here, patients with right-sided disease were more likely to achieve ETS if they were treated with panitumumab than with comparator. Median PFS and OS in patients with right-sided disease who achieved ETS were also numerically longer with panitumumab than with comparator. While intrinsic tumour sensitivity to therapy remains the main determinant of clinical outcome, ETS might therefore identify a subgroup of patients with right-sided disease who may achieve improved outcomes with panitumumab and who may therefore benefit from further treatment. Additional research is needed to help physicians identify such patients in the clinic, including those with bulky tumours and those who can be converted from nonresectable to resectable disease. The optimal treatment approach for these and other mCRC patients also needs to be better defined; for example, with respect to which treatment sequences may be best for different patients. For RAS WT patients, accumulating evidence supports the use of first-line anti-EGFR treatment, 


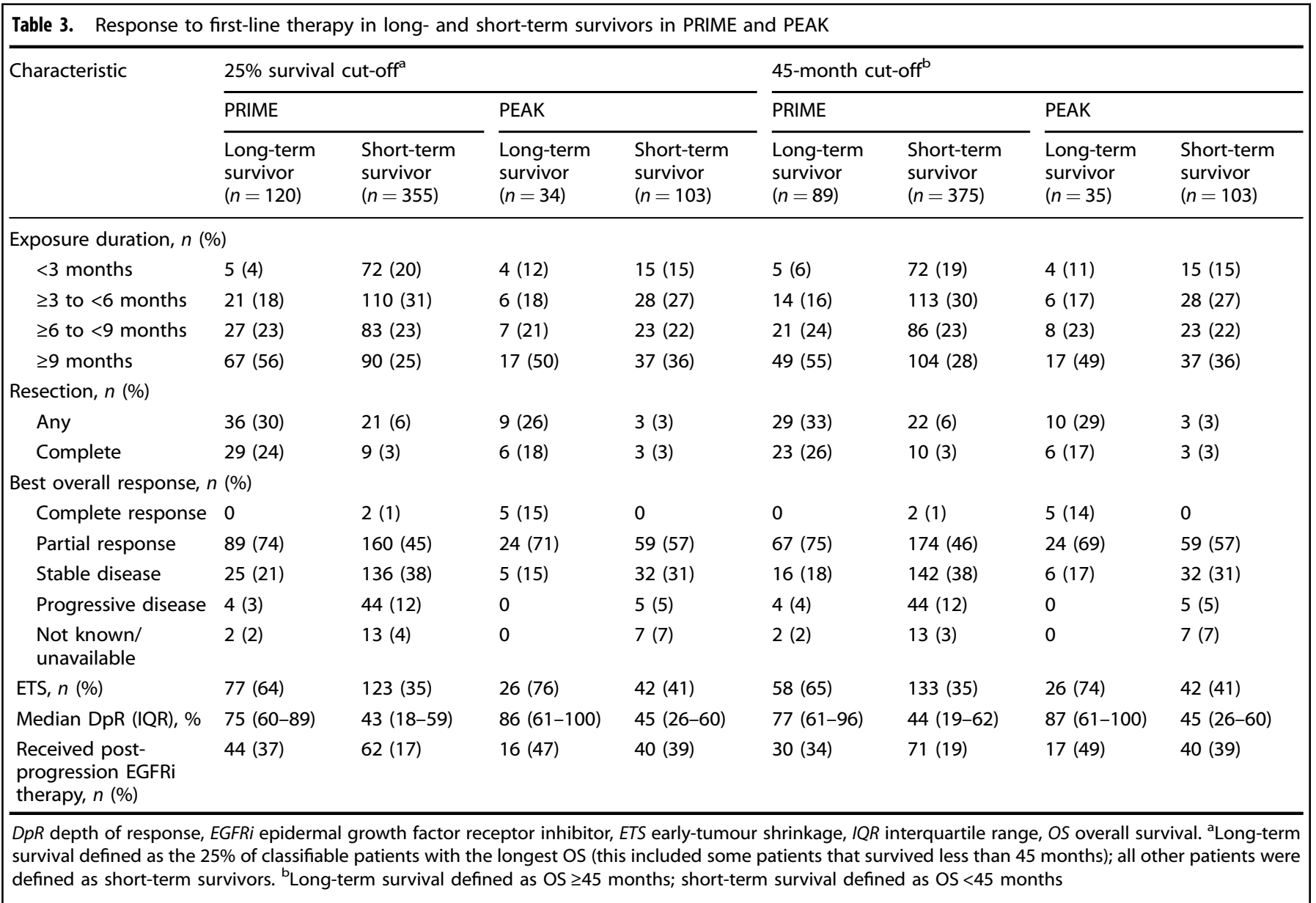

followed by anti-VEGF therapy. ${ }^{37,38}$ In this context, ETS may be useful in guiding both the decision to switch therapy and the timing of the switch.

Evidence suggests that converting non-resectable $\mathrm{mCRC}$ to resectable disease leads to better outcomes. ${ }^{39}$ In the current analyses, patients with left-sided disease were more likely to undergo tumour resection than those with right-sided disease. We also showed that in the panitumumab arms of both PRIME and PEAK, the incidence of post-progression anti-EGFR therapy was higher in patients with left- than right-sided disease. This may be because the former group of patients are more likely to have shown a good response to first-line panitumumab. In patients in the comparator arms, the incidence of post-progression anti-EGFR therapy was similar regardless of primary tumour location. The dynamic susceptibility of $\mathrm{mCRC}$ to therapeutic EGFR blockade means that it may be possible to extend survival by rechallenge with EGFR inhibitors. Anti-EGFR treatment may select for treatment-resistant subclones that are present before treatment, leading to secondary resistance of the tumour. ${ }^{40}$ Removing antiEGFR selection pressure, via a break in anti-EGFR treatment, may allow sensitive clones to become re-established, restoring tumour sensitivity. ${ }^{41-43}$ Rechallenge after a break from EGFR inhibitor treatment may thus be a viable strategy for later lines of treatment in patients with a good initial response. ${ }^{44,45}$ As an early indication of patients who are responding to treatment, ETS might therefore help identify patients suitable for rechallenge. Prospective controlled trials testing rechallenge, and attempting to identify biopsy biomarkers that can predict its efficacy, as well as the mechanisms underlying fluctuating tumour sensitivity to treatment, are currently ongoing, including A-REPEAT (NCT03311750), CHRONOS (NCT03227926) and RASINTRO (NCT03259009).

The second set of analyses reported here aimed to identify clinicopathologic characteristics beyond primary tumour location that may predict a favourable prognosis in patients with RAS WT mCRC. These analyses used 'long-term survival' as an indicator of good prognosis, defined as either patients with OS of at least 45 months (equivalent to $19 \%$ of patients in PRIME and $25 \%$ of patients in PEAK) or the $25 \%$ of patients with the longest OS. Applying either of these definitions of long-term survival in multivariate analyses, panitumumab treatment was the only factor that significantly predicted long-term survival in both the PRIME and the PEAK trials (and in a pooled analysis of both). Other characteristics that significantly predicted longterm survival in either PRIME or PEAK or both trials combined included baseline disease characteristics associated with favourable prognosis (e.g. low- or medium-risk Köhne score, liver-only metastases, ECOG performance status of 0, BRAF WT status), and left-sided disease. Some characteristics could not be modelled in the multivariate analyses due to imbalances across the subgroups, as revealed by univariate analyses. Compared with short-term survivors, long-term survivors more frequently experienced an objective response or ETS, were more likely to have had a resection and had a greater $\mathrm{DpR}$. To our knowledge this is the first reported analysis to compare characteristics of long- and short-term survivors among a cohort of $\mathrm{mCRC}$ patients treated with anti-EGFR therapy. Of note, however, ETS has been shown to be associated with longer PFS and OS in patients with 
Table 4. Independent predictors of long-term overall survival in multivariate analysis in PRIME and PEAK (45-month cut-off for longterm survival)

\begin{tabular}{|c|c|}
\hline & Odds ratio $(95 \% \mathrm{C}$ \\
\hline \multicolumn{2}{|l|}{ PRIME } \\
\hline \multicolumn{2}{|l|}{ Köhne score } \\
\hline Low risk vs. high risk & $3.00(0.33-27.49)$ \\
\hline Medium risk vs. high risk & $6.26(2.15-18.29)$ \\
\hline $\begin{array}{l}\text { Treatment: FOLFOX4 vs. panitumumab + } \\
\text { FOLFOX4 }\end{array}$ & $0.50(0.30-0.83)$ \\
\hline \multicolumn{2}{|l|}{ Sites of metastases } \\
\hline Liver + other vs. liver only & $0.28(0.04-2.26)$ \\
\hline Other only vs. liver only & $0.11(0.01-0.85)$ \\
\hline \multicolumn{2}{|l|}{ BRAF status } \\
\hline Mutant vs. wild-type & $0.08(0.01-0.63)$ \\
\hline Missing vs. wild-type & $1.58(0.37-6.80)$ \\
\hline $\begin{array}{l}\text { Region: Rest of the world vs. Western Europe, } \\
\text { Canada or Australia }\end{array}$ & $0.56(0.32-0.96)$ \\
\hline Age ( $\geq 65$ years): No vs. yes & $1.69(0.98-2.91)$ \\
\hline \multicolumn{2}{|l|}{ Stage } \\
\hline I-III vs. IV & $2.37(1.29-4.36)$ \\
\hline Missing vs. IV & $1.04(0.20-5.46)$ \\
\hline \multicolumn{2}{|l|}{ PEAK } \\
\hline $\begin{array}{l}\text { Treatment: mFOLFOX } 6 \text { + bevacizumab vs. } \\
\text { mFOLFOX } 6 \text { + panitumumab }\end{array}$ & $0.36(0.15-0.89)$ \\
\hline Sex: Female vs. male & $2.93(1.17-7.34)$ \\
\hline \multicolumn{2}{|l|}{ Primary tumour location } \\
\hline Right vs. left & $0.16(0.04-0.63)$ \\
\hline Unknown vs. left & $0.25(0.05-1.26)$ \\
\hline Prior adjuvant chemotherapy: No vs. yes & $0.37(0.13-1.08)$ \\
\hline ECOG performance status: 1 vs. 0 & $0.29(0.11-0.81)$ \\
\hline \multicolumn{2}{|l|}{ PRIME and PEAK } \\
\hline \multicolumn{2}{|l|}{ Köhne score } \\
\hline Low risk vs. high risk & $7.85(2.93-21.03)$ \\
\hline Medium risk vs. high risk & $5.61(2.18-14.42)$ \\
\hline Treatment: Comparator vs. panitumumab & $0.51(0.33-0.77)$ \\
\hline \multicolumn{2}{|l|}{ BRAF status } \\
\hline Mutant vs. wild-type & $0.07(0.01-0.54)$ \\
\hline Missing vs. wild-type & $0.94(0.24-3.73)$ \\
\hline \multicolumn{2}{|l|}{ Side of tumour } \\
\hline Right vs. left & $0.49(0.25-0.94)$ \\
\hline Unknown vs. left & $0.48(0.25-0.94)$ \\
\hline
\end{tabular}

Cl confidence interval, ECOG Eastern Cooperative Oncology Group, mFOLFOX6 modified FOLFOX6. ${ }^{\mathrm{a}} \mathrm{A}$ stepwise model-building procedure was used with a $10 \%$ significance level for a covariate to enter or remain in the model. The table shows all covariates that were significant at the $10 \%$ significance level in the final model. An odds ratio $>1$ indicates that longterm survival is more likely for the first parameter listed, while an odds ratio $<1$ indicates that long-term survival is more likely for the second parameter listed

RAS WT mCRC treated with cetuximab. ${ }^{25}$ Further to this, a retrospective analysis of three trials in RAS WT MCRC patients found that ETS and DpR were associated with improved survival. ${ }^{24}$ However, these studies were not designed to compare long- and short-term survivors.

The analyses reported here have provided further insights into the impact of primary tumour location and panitumumab treatment on outcomes in PRIME and PEAK, and on factors beyond left-sided disease that may predict a good prognosis in patients with RAS WT mCRC. A limitation of our analyses is that they were post-hoc and unplanned. With respect to ETS and DpR specifically, these endpoints were not in use when the trials were designed $\sim 10$ years ago, and thus they were not included as prespecified endpoints in the trial protocols. ETS and DpR are now, however, used increasingly in $\mathrm{mCRC}$ as early indicators of treatment efficacy. ${ }^{25-27}$ A further limitation is that differences in OS were observed between the full study populations of PRIME and PEAK, which may reflect differences in patient characteristics between the two studies (and/or greater data variability in smaller studies such as PEAK or differences in treatment sequencing or use of anti-angiogenic (bevacizumab) therapy). In this respect and due to the differences in trial methodology and the number of patients recruited to each trial, pooled data should be cautiously interpreted. In addition, some patients in the full study populations were omitted from these analyses since data on, for example, tumour mutation status or primary tumour location were not available, or in the case of the long-term survivor analyses, because survival data were censored before the cut-off date. Finally, some of the subgroups analysed, especially those deriving from the PEAK trial and those containing patients with right-sided disease, contained very small numbers of patients that may have impacted on validity of these analyses.

In conclusion, the additional analyses of PRIME and PEAK reported here have shown that first-line panitumumab plus chemotherapy resulted in a higher rate of ETS and a higher DpR compared with first-line chemotherapy (with or without bevacizumab) in RAS WT patients with left-sided primary tumours. Pooled analyses also confirmed that panitumumab improved survival outcomes, including PFS and OS, vs. comparator in these patients. In patients with right-sided disease, data were difficult to interpret due to the low patient numbers in this subgroup, yet may indicate that ETS could be used to identify a subgroup of patients with right-sided disease who may achieve improved outcomes with panitumumab therapy. Panitumumab treatment significantly predicted long-term survival in both PRIME and PEAK.

\section{ACKNOWLEDGEMENTS}

We thank all patients, their families and all investigators involved in the PRIME and PEAK studies. Medical writing support (including development of a draft outline and subsequent drafts in consultation with the authors, assembling tables and figures, collating author comments, copyediting, fact checking and referencing) was provided by Louisa Lyon, DPhil and Louise Niven, DPhil at Aspire Scientific (Bollington, UK), and funded by Amgen (Europe) GmbH (Zug, Switzerland).

\section{AUTHOR CONTRIBUTIONS}

R.K. and P.B. were responsible for conception and design of the analyses. T.P. and J.-L.C. contributed to the design of the analyses. M.P., T.P., J.T., F.R., J.-L.C. and S.S. contributed to patient data collection. RK performed the data analyses. All authors contributed to the interpretation of the data, the preparation and revision of the manuscript, and approved the final version.

\section{ADDITIONAL INFORMATION}

Supplementary information is available for this paper at https://doi.org/10.1038/ s41416-018-0165-z.

Competing interests: M.P. has received research funding from Amgen, Roche and Sirtex, and honoraria from Amgen, Merck Serono, Roche, Sanofi Aventis, Servier and Sirtex. T.P. has acted on advisory boards for Amgen, Merck Serono and Roche, and has received travel support from Amgen. J.T. has acted in consultancy and/or advisory roles for, and received honoraria from Amgen, Baxalta, Celgene, Eli Lilly, Merck, Roche, Sanofi, Servier and Sirtex. Michael Geissler has received research funding and acted in consultancy/advisory roles for Amgen, Bayer, Merck, Roche and 
Sanofi. F.R. has received research funding from and/or acted on advisory boards for Amgen, Bayer, Celgene, Eli Lilly, Merck Serono, Merck Sharp \& Dohme, Roche, Servier and Sanofi. J.-L.C. is a consultant/advisor for Amgen and Roche and has received institutional funding from Amgen, Novartis and Roche. G.P. has received research funding and advisory consulting fees from Amgen, Astra Zeneca, Boehringer, Bristol Myers Squibb, Lilly, Merck, MSD and Roche. R.K. is an employee of Amgen Ltd and owns restricted shares in Amgen. P.B. is an employee of Amgen (Europe) GmbH and owns shares in Amgen. S.S. is a member of advisory boards for Amgen, Bayer, Celgene, Eli Lilly, Ignyta, Merck, Merrimack, Novartis, Roche and Sanofi.

Consent to participate: All patients provided informed consent.

Ethics approval: The PRIME and PEAK studies were conducted in accordance with the Declaration of Helsinki. Study protocols were approved by an independent ethics committee at each study center.

Funding: The PRIME (NCT00364013) and PEAK (NCT00819780) studies were supported by Amgen Inc.

Publisher's note: Springer Nature remains neutral with regard to jurisdictional claims in published maps and institutional affiliations.

\section{REFERENCES}

1. Ferlay, J. et al. Cancer incidence and mortality worldwide: sources, methods and major patterns in GLOBOCAN 2012. Int J. Cancer 136, E359-E386 (2015).

2. Van Cutsem, E., Cervantes, A., Nordlinger, B., Arnold, D. \& Group, E. G. W. Metastatic colorectal cancer: ESMO Clinical Practice Guidelines for diagnosis, treatment and follow-up. Ann. Oncol. 25(Suppl 3), iii1-iii9 (2014).

3. National Comprehensive Cancer Network. Clinical Practice Guidelines in Oncology: Colon Cancer Version 2.2018. https://www.nccn.org/professionals/ physician_gls/default.aspx. Accessed May 2018.

4. Van Cutsem, E. et al. ESMO consensus guidelines for the management of patients with metastatic colorectal cancer. Ann. Oncol. 27, 1386-1422 (2016).

5. Douillard, J. Y. et al. Randomized, phase III trial of panitumumab with infusional fluorouracil, leucovorin, and oxaliplatin (FOLFOX4) versus FOLFOX4 alone as firstline treatment in patients with previously untreated metastatic colorectal cancer: the PRIME study. J. Clin. Oncol. 28, 4697-4705 (2010).

6. Bokemeyer, C. et al. Efficacy according to biomarker status of cetuximab plus FOLFOX-4 as first-line treatment for metastatic colorectal cancer: the OPUS study. Ann. Oncol. 22, 1535-1546 (2011).

7. Clarke, C. N. \& Kopetz, E. S. BRAF mutant colorectal cancer as a distinct subset of colorectal cancer: clinical characteristics, clinical behavior, and response to targeted therapies. J. Gastrointest. Oncol. 6, 660-667 (2015).

8. Pietrantonio, F. et al. Predictive role of BRAF mutations in patients with advanced colorectal cancer receiving cetuximab and panitumumab: a meta-analysis. Eur. J. Cancer 51, 587-594 (2015).

9. Rowland, A. et al. Meta-analysis of BRAF mutation as a predictive biomarker of benefit from anti-EGFR monoclonal antibody therapy for RAS wild-type metastatic colorectal cancer. Br. J. Cancer 112, 1888-1894 (2015).

10. Jones, J. C. et al. Non-V600BRAF mutations define a clinically distinct molecular subtype of metastatic colorectal cancer. J. Clin. Oncol. 35, 2624-2630 (2017).

11. Seligmann, J. F. et al. Association of tumor HER3 messenger RNA expression with panitumumab efficacy in advanced colorectal cancer. JAMA Oncol. 4, 564-568 (2018).

12. Sartore-Bianchi, A., Marsoni, S. \& Siena, S. Human epidermal growth factor receptor 2 as a molecular biomarker for metastatic colorectal cancer. JAMA Oncol. 4, 19-20 (2018).

13. Scartozzi, M. et al. Analysis of HER-3, insulin growth factor-1, nuclear factor-kB and epidermal growth factor receptor gene copy number in the prediction of clinical outcome for K-RAS wild-type colorectal cancer patients receiving irinotecan-cetuximab. Ann. Oncol. 23, 1706-1712 (2012).

14. Boeckx, N. et al. Primary tumor sidedness has an impact on prognosis and treatment outcome in metastatic colorectal cancer: results from two randomized first-line panitumumab studies. Ann. Oncol. 28, 1862-1868 (2017).

15. Loupakis, F. et al. Primary tumor location as a prognostic factor in metastatic colorectal cancer. J. Natl. Cancer Inst. 107, dju427 (2015).

16. Tejpar, S., Stintzing, S. \& Ciardiello, F. Prognostic and predictive relevance of primary tumor location in patients with RAS wild-type metastatic colorectal cancer: Retrospective analyses of the CRYSTAL and FIRE-3 trials. JAMA Oncol. 3, 194-201 (2017).

17. Petrelli, F., Tomasello, G. \& Borgonovo, K. Prognostic survival associated with leftsided vs right-sided colon cancer: a systematic review and meta-analysis. JAMA Oncol. 3, 211-219 (2017).
18. Stintzing, S., Tejpar, S., Gibbs, P., Thiebach, L. \& Lenz, H. J. Understanding the role of primary tumour localisation in colorectal cancer treatment and outcomes. Eur. J. Cancer 84, 69-80 (2017).

19. Boeckx, N. et al. The predictive value of primary tumor location in patients with metastatic colorectal cancer: a systematic review. Crit. Rev. Oncol. Hematol. 121, 1-10 (2018).

20. Sartore-Bianchi, A. et al. Dual-targeted therapy with trastuzumab and lapatinib in treatment-refractory, KRAS codon 12/13 wild-type, HER2-positive metastatic colorectal cancer (HERACLES): a proof-of-concept, multicentre, open-label, phase 2 trial. Lancet Oncol. 17, 738-746 (2016).

21. Guinney, J. et al. The consensus molecular subtypes of colorectal cancer. Nat. Med. 21, 1350-1356 (2015).

22. Arnold, D. et al. Prognostic and predictive value of primary tumour side in patients with RAS wild-type metastatic colorectal cancer treated with chemotherapy and EGFR directed antibodies in six randomised trials. Ann. Oncol. 28, 1713-1729 (2017).

23. Holch, J. W., Ricard, I., Stintzing, S., Modest, D. P. \& Heinemann, V. The relevance of primary tumour location in patients with metastatic colorectal cancer: A metaanalysis of first-line clinical trials. Eur. J. Cancer 70, 87-98 (2017).

24. Taieb, J. et al. Exploratory analyses assessing the impact of early tumour shrinkage and depth of response on survival outcomes in patients with RAS wildtype metastatic colorectal cancer receiving treatment in 3 randomised panitumumab trials. J. Cancer Res Clin. Oncol. 144, 321-335 (2018).

25. Piessevaux, H. et al. Use of early tumor shrinkage to predict long-term outcome in metastatic colorectal cancer treated with cetuximab. J. Clin. Oncol. 31, 3764-3775 (2013).

26. Douillard, J. Y. et al. Impact of early tumour shrinkage and resection on outcomes in patients with wild-type RAS metastatic colorectal cancer. Eur. J. Cancer 51, 1231-1242 (2015).

27. Heinemann, V. et al. Early tumour shrinkage (ETS) and depth of response (DpR) in the treatment of patients with metastatic colorectal cancer (mCRC). Eur J Cancer 32, 1927-1936 (2015).

28. Peeters M., et al. Impact of primary tumour location on response and resection outcomes in patients with metastatic colorectal cancer (mCRC) undergoing firstline treatment (abstract P-321). Ann Oncol. 28, 2017 https://doi.org/10.1093/ annonc/mdx261.318

29. Köhne C.-H., et al. Characteristics and response outcomes of long-term survivors in studies of first-line panitumumab for metastatic colorectal cancer (abstract $\mathrm{P}$ 319). Ann Oncol. 28, 2017 https://doi.org/10.1093/annonc/mdx261.316

30. Douillard, J. Y. et al. Panitumumab-FOLFOX4 treatment and RAS mutations in colorectal cancer. N. Engl. J. Med. 369, 1023-1034 (2013).

31. Schwartzberg, L. S. et al. PEAK: a randomized, multicenter phase II study of panitumumab plus modified fluorouracil, leucovorin, and oxaliplatin (mFOLFOX6) or bevacizumab plus mFOLFOX6 in patients with previously untreated, unresectable, wild-type KRAS exon 2 metastatic colorectal cancer. J. Clin. Oncol. 32, 2240-2247 (2014).

32. Price, T. J. et al. Update on optimal treatment for metastatic colorectal cancer from the ACTG/AGITG expert meeting: ECCO 2015. Expert Rev. Anticancer Ther. 16, 557-571 (2016).

33. Vogel, A., Hofheinz, R. D., Kubicka, S. \& Arnold, D. Treatment decisions in metastatic colorectal cancer - beyond first and second line combination therapies. Cancer Treat. Rev. 59, 54-60 (2017).

34. Kohne, C. H. et al. Clinical determinants of survival in patients with 5-fluorouracilbased treatment for metastatic colorectal cancer: results of a multivariate analysis of 3825 patients. Ann. Oncol. 13, 308-317 (2002).

35. Folprecht, G. et al. Tumour response and secondary resectability of colorectal liver metastases following neoadjuvant chemotherapy with cetuximab: the CELIM randomised phase 2 trial. Lancet Oncol. 11, 38-47 (2010).

36. Geissler M., et al. mFOLFOXIRI + panitumumab versus FOLFOXIRI as first-line treatment in patients with RAS wild-type metastatic colorectal cancer $\mathrm{m}(\mathrm{CRC})$ : a randomized phase II VOLFI trial of the AIO (AIO-KRK0109) (abstract 475O). Ann. Oncol. 28, 2017; https://doi.org/10.1093/annonc/mdx393.002

37. Sobrero, A. et al. Impact of sequence of biologic therapies on overall survival (OS) in patients with RAS wild-type (WT) metastatic colorectal carcinoma (mCRC): an exploratory, retrospective pooled analysis (abstract P-149). Ann. Oncol. 27, ii44 (2016).

38. Zaniboni, A. \& Formica, V. The Best. First. Anti-EGFR before anti-VEGF, in the firstline treatment of RAS wild-type metastatic colorectal cancer: from bench to bedside. Cancer Chemother. Pharmacol. 78, 233-244 (2016).

39. Folprecht, G. et al. Survival of patients with initially unresectable colorectal liver metastases treated with FOLFOX/cetuximab or FOLFIRI/cetuximab in a multidisciplinary concept (CELIM study). Ann. Oncol. 25, 1018-1025 (2014).

40. Misale, S. et al. Emergence of KRAS mutations and acquired resistance to antiEGFR therapy in colorectal cancer. Nature 486, 532-536 (2012). 
41. Siravegna, G. et al. Monitoring clonal evolution and resistance to EGFR blockade in the blood of metastatic colorectal cancer patients. Nat. Med. 21, 795-801 (2015).

42. Santini, D. et al. Cetuximab rechallenge in metastatic colorectal cancer patients: how to come away from acquired resistance? Ann. Oncol. 23, 2313-2318 (2012).

43. Ciardiello, F. et al. Cetuximab continuation after first progression in metastatic colorectal cancer (CAPRI-GOIM): a randomized phase II trial of FOLFOX plus cetuximab versus FOLFOX. Ann. Oncol. 27, 1055-1061 (2016).

44. Siena S., et al. Rechallenge with EGFR inhibitors in patients with metastatic colorectal cancer: effect on outcomes (abstract P-320). Ann. Oncol. 28, 2017; https:// doi.org/10.1093/annonc/mdx261.317

45. Tonini, G., Imperatori, M., Vincenzi, B., Frezza, A. M. \& Santini, D. Rechallenge therapy and treatment holiday: different strategies in management of metastatic colorectal cancer. J. Exp. Clin. Cancer Res. 32, 92 (2013).
Open Access This article is licensed under a Creative Commons Attribution 4.0 International License, which permits use, sharing, adaptation, distribution and reproduction in any medium or format, as long as you give appropriate credit to the original author(s) and the source, provide a link to the Creative Commons license, and indicate if changes were made. The images or other third party material in this article are included in the article's Creative Commons license, unless indicated otherwise in a credit line to the material. If material is not included in the article's Creative Commons license and your intended use is not permitted by statutory regulation or exceeds the permitted use, you will need to obtain permission directly from the copyright holder. To view a copy of this license, visit http://creativecommons. org/licenses/by/4.0/.

(c) The Author(s) 2018 